\title{
新型吡唑联吡咯类杂环化合物的合成及杀菌活性
}

\author{
王子时 ${ }^{a}$ 叶汉韬 ${ }^{a}$ 肖 丹 ${ }^{a}$ 马东升 ${ }^{*, a}$ 高金胜 $^{a, b}$ 于颖慧 $^{a}$ \\ 侯广峰*, 间鹏飞 $a$ \\ ( ${ }^{a}$ 黑龙江大学化学化工与材料学院 哈尔滨 150080) \\ ( ${ }^{b}$ 黑龙江大学农药工程中心 哈尔滨 150080)
}

\begin{abstract}
摘要 为寻找具有更高生物活性、更环保的新农药杀菌剂, 基于杀菌剂氟咯菌腈设计并合成了 10 个新型吡唑联吡咯类 杂环化合物, 以吡唑环代替氟咯菌腈上原有的苯环, 以期提高其杀菌活性. 采用 ${ }^{1} \mathrm{H}$ NMR, FTIR, 单晶 X 射线衍射、元 素分析和熔点测定等测试手段对目标化合物及其中间体的结构进行了表征与确认，并通过挥发法培养得到了 6 个目标 化合物的单晶. 还对其中 6 个化合物进行了生物活性测试, 测试结果表明各化合物对水稻纹枯病、黄瓜灰霉病、黄瓜 霜霉病均表现出一定的抑菌效果, 可作为先导化合物对其结构进行深入的研究, 为新农药杀菌剂的开发提供了理论支 持.
\end{abstract}

关键词 氟咯菌腈; 吡唑联吡咯类化合物; 合成; 反应条件; 生物活性测试

\section{Synthesis and Fungicidal Activity of New Pyrrolpyrazole Compounds}

\author{
Wang $\mathrm{Zishi}^{a}$ \\ Ye, Hantao ${ }^{a}$ \\ Xiao, $\operatorname{Dan}^{a}$ \\ Ma, Dongsheng*,a \\ Gao, Jinsheng ${ }^{a, b}$ \\ Yu, Yinghui ${ }^{a}$ \\ Hou, Guangfeng*,b Yan, Pengfei ${ }^{a}$ \\ $\left({ }^{a}\right.$ School of Chemistry and Materials Science, Heilongjiang University, Harbin 150080) \\ $\left({ }^{b}\right.$ Engineering Research Center of Pesticide of Heilongjiang University, Heilongjiang University, Harbin 150080)
}

\begin{abstract}
With the aim of obtaining new potential fungicide candidates with higher biological activities and environmental safety, ten new pyrrolpyrazole compounds based on the structures of fludioxonil were designed and synthesized. The benzene ring in original fludioxonil structure is replaced by pyrazole ring to improve its bactericidal activity. All the structures of target products and their intermediates are characterized and confirmed by ${ }^{1} \mathrm{H}$ NMR, FTIR, single crystal diffraction, HPLC, elemental analysis and melting point determination. Six single crystals of final products are also achieved by volatilization under room temperature. Biological activity tests are conducted for six compounds. The results show that each compound exhibits certain antibacterial activity toward rhizoctonia, botrytis cinerea and cucumber downy mildew, which provides valuable information for further development of new pesticides fungicides.

Keywords fludioxonil; pyrrolpyrazole compound; synthesis; reaction condition; biological activity test
\end{abstract}

随着世界农药的不断发展, 新型含氮杂环农药已成 为人们研究的热点之一. 其中含吡唑环和吡咯环 ${ }^{[1 \sim 3]}$ 的 化合物作为含氮杂环化合物中的主要代表, 其衍生物均 表现出广泛的杀菌、杀虫、除草等活性. 到目前为止, 许 多新型农药品种已被开发出来, 如吡唑类杀菌剂吡噻菌 胺、吡唑类杀虫剂吡螨胺、吡咯类杀菌剂氟咯菌腈等. 氟 咯菌腈 ${ }^{[4,5]}$ 是由瑞士先正达公司开发的一种非内吸性的
新型杀菌剂，以其高效、环保、持效期长的特点，一经 上市便被用作种子处理剂而广泛应用在果树、蔬菜、谷 物等农作物病害的防治上, 效果显著, 具有广阔的市场 前景.

从结构上来看氟咯菌腈是以苯环和吡咯环为主要 结构的杀菌剂, 其主要活性基团为苯环上取代的氟原子 和氰基吡咯结构. 本课题组针对氟咯菌腈的结构, 前期

* E-mail: hougf@hlju.edu.cn

Received April 3, 2014; revised May 7, 2014; published online June 9, 2014.

Project supported by the National Natural Science Foundation of China (Nos. 21371052 and 21272061) and the Scientific Research Fund of Heilongjiang Provincial Education Department (No. 12531512).

国家自然科学基金(Nos. 21371052, 21272061)及黑龙江省教育厅科学研究基金(No. 12531512)资助项目. 
对其进行了系统的研究, 在保留其氰基吡咯结构的基础 上，对其苯环进行了结构的修饰，合成出一系列的氟咯 菌腈衍生物 ${ }^{[6]}$. 但从结构角度来说, 所合成的化合物结 构仍然为苯环和吡咯环为主体, 且从修饰基团来看, 氯 原子、甲氧基、硝基的引入，对其生物活性的提高没有 显著地影响. 随着研究的深入, 本文选用具有良好生物 活性的吡唑环代替氟咯菌腈原有的苯环结构, 构筑一类 新型的双含氮杂环化合物. 吡唑环作为一种应用广泛的 结构单元，通过亚甲基、砜基 ${ }^{[7]}$ 等基团与含氮杂环相连 接, 或与含氮杂环直接相连接得到的双含氮杂环化合 物, 均表现出良好的生物活性 ${ }^{[8 \sim 10]}$.

本文基于课题组对吡咯类杀菌剂氟咯菌腈详细研 究, 以吡唑环代替其原有的苯环, 最终合成了 10 个未被 文献报道的新型吡唑联吡咯类杂环化合物. 同时采用 ${ }^{1} \mathrm{H}$ NMR, FTIR、单晶 X 射线衍射、元素分析和熔点测 定等测试手段对目标化合物及其中间体的结构进行了 表征与确认. 此外, 本文还对其中 6 个吡唑联吡咯类化 合物进行了生物活性测试, 测试结果显示, 在浓度为 $200 \mathrm{mg} / \mathrm{L}$ 时, 各化合物对水稻纹枯病、黄瓜灰霉病、黄 瓜霜霉病均表现出一定的抑制效果. 目标化合物的合成 路线如 Scheme 1 所示.

\section{1 结果与讨论}

\section{1 合成}

TosMIC-对甲苯磺酰甲基异腈(4)的合成是以对甲 苯磺酰氯为起始原料, 经两步反应制备得到的. 合成 TosMIC 的反应机理为, 在三乙胺作碱的条件下，中间 体 3 上酰胺基上的 $\mathrm{N}$ 原子脱掉 $\mathrm{H}$ 离子生成氮负离子，进 而构型转变为氧负离子, 然后与三氯氧磷反应得到一个 氯代亚甲基铵盐, 最后脱水得到最终产物 $\mathbf{4}^{[11]}$.

1,3-二甲基-5-吡唑酮(7a) 是由乙酰乙酸乙酯与甲基 肼(40\%)关环得到 ${ }^{[12 ~ 14]}$, 由于酯羰基的活泼性没有羰基 的活泼性高, 且伯胺比仲胺的亲和性更强, 因此经过脱 水、再脱乙醇的过程, 得到唯一的产物. 同时为了加快 反应速率，选择乙醇为溶剂效果最佳.

合成 5-氯-1,3-二甲基-1H-吡唑-4-甲醛(8a) 的反应是 一类经典的人名反应，即 Vilsmeier-Haack-Arnold 反应 (简称: VHA 反应) ${ }^{[15 ~ 17]}$, 以 $\mathrm{DMF}$ 与 $\mathrm{POCl}_{3}$ 反应先形成两 种稳定的活性加合中间体，即氯代亚甲基铵盐的平衡 (VHA 试剂), 然后 VHA 试剂亲电进攻 $7 \mathbf{a}$ 上表现出弱碱 性的碳氧双键，进而生成双亚甲基铵盐. 加热脱去 DMF 后，再通过水解生成 8a. 上述反应可称为氯代甲酰化反

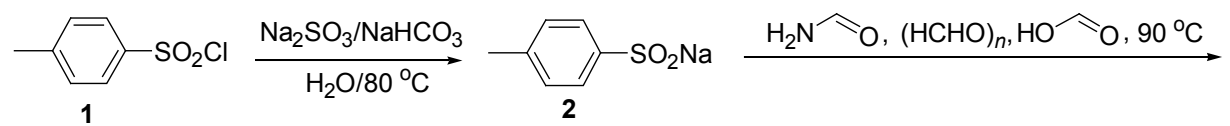

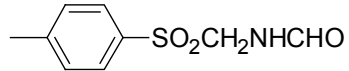

3

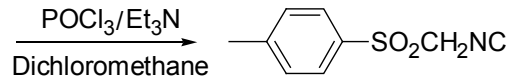

4

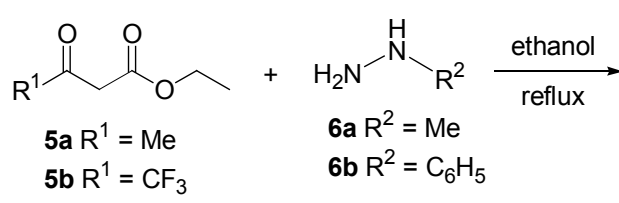

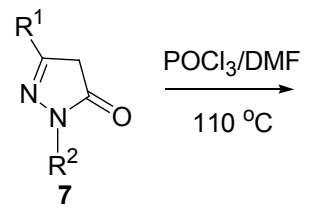
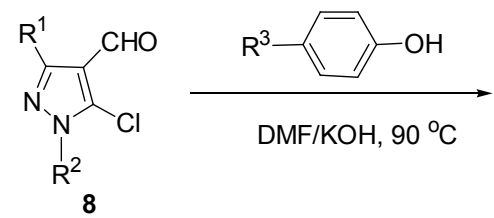

$7 a, 8 a R^{1}=M e, R^{2}=M e ; 7 b, 8 b R^{1}=C F_{3}, R^{2}=M e ; 7 c, 8 c R^{1}=M e, R^{2}=C_{6} H_{5}$

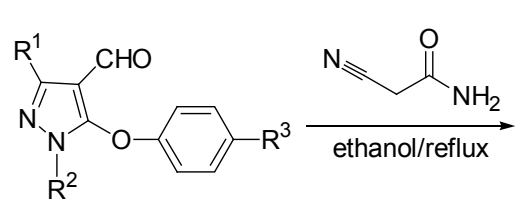

9

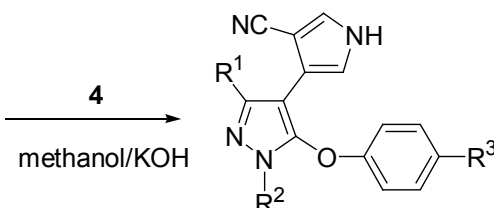

11

$$
\begin{array}{ll}
\text { 9a } \sim 11 a R^{1}=M e, R^{2}=M e, R^{3}=F ; & 9 f \sim 11 f R^{1}=C_{3}, R^{2}=M e, R^{3}=F ; \\
\text { 9b 11b } R^{1}=M e, R^{2}=M e, R^{3}=M e ; & 9 g \sim 11 g R^{1}=C_{3}, R^{2}=M e, R^{3}=M e ; \\
\text { 9c } \sim 11 c R^{1}=M e, R^{2}=M e, R^{3}=\left(C_{3}\right)_{3} ; & 9 h \sim 11 h R^{1}=C_{3}, R^{2}=M e, R^{3}=\left(C_{3}\right)_{3} ; \\
\text { 9d 11d } R^{1}=M e, R^{2}=M e, R^{3}=\mathrm{OCH}_{3} ; & 9 i \sim 11 i R^{1}=C_{3}, R^{2}=M e, R^{3}=\mathrm{OCH}_{3} ; \\
9 e \sim 11 e R^{1}=C_{3}, R^{2}=M e, R^{3}=H ; & 9 j \sim 11 j R^{1}=M e, R^{2}=C_{6} H_{5}, R^{3}=M e
\end{array}
$$

Scheme 1 
应，通过此反应可在吡唑酮的 5-位引入一个氯原子，同 时在 4-位上引入一个醛基. 应注意的是, 在该反应过程 中需严格控制三氯氧磷的滴加速度, 保持温度在 $20 \sim$ $30{ }^{\circ} \mathrm{C}$ 之间, 并且升温后的反应时间也不宜过长, 一般 在 $6 \mathrm{~h}$ 左右, 因为反应时间过长会导致产物的收率降低.

合成中间体 9 是由中间体 8 和苯酚类化合物在碱性 条件下发生的亲核取代反应而制备的 ${ }^{[18,19]}$. 通过实验发 现，苯酚类化合物酚羟基的对位基团对反应的影响较 大. 在 DMF 为溶剂, 高温加热的条件下, 酚羟基的对位 为吸电子基团的化合物不与 $\mathbf{8}$ 发生亲核取代反应, 如对 硝基苯酚、对三氟甲基苯酚等; 而酚羟基的对位为供电 子基团的化合物则与 8 发生亲核取代反应, 其产品收率 较好, 纯度较高, 如对甲酚、对甲氧基苯酚等. 中间体 10 是在乙醇作溶剂, 碱性条件下回流, 通过 Knoevenagel 缩合得到的 ${ }^{[20]}$, 其中选用三乙胺作为碱, 催化效果好, 缩短了反应时间, 后处理操作简便.

目标化合物 11 是碱性条件下制备的, 首先碱性条 件使化合物 4 亚甲基上的碳原子形成碳负离子, 而后与 化合物 10 上的碳碳双键发生 Michael 加成反应, 再发生 关环反应得到目标化合物 $\mathbf{1 1}^{[21,22]}$, 实验过程中溶剂的类 别、碱和化合物 $\mathbf{4}$ 的用量对实验结果都有较大的影响.

\section{2 图谱分析}

目标化合物的红外光谱分析，从数据上看 $\mathbf{1 1 a} \sim 11 \mathbf{j}$ 在 $3000,2200,1680$ 和 $1560 \mathrm{~cm}^{-1}$ 附近有特征吸收峰; 目 标化合物的 ${ }^{1} \mathrm{H}$ NMR 核磁共振谱图分析, 11a 11d 吡唑 环上的甲基, 其化学位移一般在 $\delta 2.0 \sim 3.9$ 之间, 且均 为单峰; $11 \mathrm{a} \sim 11 \mathrm{j}$ 吡咯环上 2 -位和 5-位上分别存在着两 个氢, 其中 2-位上的 $\mathrm{H}$ 受吡咯环的共轭效应和 3-位上氧 基的吸电子作用, 化学位移向低场移动, 且一般为单峰; 苯环上的 H 化学位移一般在 $\delta 7.0$ 左右, 且多为多重峰, 具体位置归属需根据化合物的结构来判断.

通过单晶 $\mathrm{X}$ 射线衍射可以表明, 化合物 11a 是结晶 在正交晶系的 $P 2_{1} / c$ 空间群(图 1). 其不对称单元图可以 很好的表征该化合物的分子结构. 在其不对称单元是由 一个完整的 11a 分子组成. 此外, 通过单晶结构, 我们 还可以看出化合物 11a 中的 3 个芳香环并不在同一平面 上, 三者之间呈现一定的夹角: 苯环与吡咯环之间的二 面角为 $63.1(1)^{\circ}$, 苯环与吡唑环之间的二面角为 $89.2(1)^{\circ}$, 吡唑与吡咯之间的二面角为 $47.6(1)^{\circ}$. 相邻的 两个 $11 \mathrm{a}$ 分子通过 $\mathrm{N}(3)-\mathrm{H}(31) \cdots \mathrm{N}(2)$ 氢键相连, 形成一 条沿 [010]方向的标准直线链状结构 $[\mathrm{N}(2) \mathrm{N}(2) \mathrm{N}(2)=$ $180^{\circ}$.

化合物 11b, 11c, 11e, 11g, 11h 的分子结构与 $11 \mathrm{a}$ 相 似, 其具体的单晶数据参数和单分子结构图在辅助材料 中有详细说明.

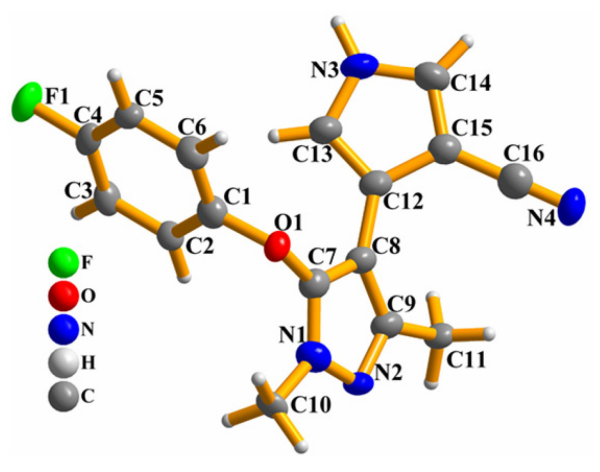

图 $111 \mathrm{a}$ 的单分子结构

Figure 1 A single molecular structure of compound 11a

\section{3 生物活性}

参照创制农药生物活性评价 $\mathrm{SOP}$ (杀菌剂卷)标准, 使用活体盆栽法, 以杀菌剂氟咯菌腈为参照物, 对本文 合成的其中目标化合物 11a, 11b, 11c, 11f, 11g, 11h 分别 对水稻纹枯病、黄瓜灰霉病、黄瓜霜霉病、黄瓜白粉病 四种菌体的抑菌活性进行普篮测试, 测试结果见表 1.

表 1 吡唑联吡咯类化合物的杀菌活性测试结果 ${ }^{a}$

Table 1 Bactericidal activity test results of the pyrrolpyrazole compounds

\begin{tabular}{ccccc}
\hline Compd. & 水稻纹枯病 & 黄瓜灰霉病 & 黄瓜霜霉病 & 黄瓜白粉病 \\
\hline $\mathbf{1 1 a}$ & 21.40 & 1.41 & 40 & 0 \\
$\mathbf{1 1 b}$ & 11.93 & 2.82 & 0 & 0 \\
$\mathbf{1 1}$ & 43.70 & 30 & 30 & 0 \\
$\mathbf{1 1 f}$ & 18.06 & 1.41 & 0 & 0 \\
$\mathbf{1 1 g}$ & 0 & 0 & 15 & 0 \\
$\mathbf{1 1 h}$ & 0 & 0 & 20 & 0 \\
氟咯菌腈 & 100 & 100 & 30 & 20 \\
\hline
\end{tabular}

a 图中数字为百分数, 特指对对应病菌的防效(\%); 测试浓度为 $200 \mathrm{mg} / \mathrm{L}$.

杀菌活性实验结果表明, 在 $200 \mathrm{mg} / \mathrm{L}$ 时不同化合 物显示出不同的杀菌活性, 其中 11a, 11c 对黄瓜霜霉病 抑制率分别为 $40 \%, 30 \%$, 其抑菌效果达到甚至超过了 参照物所体现出的抑菌效果. 因此基本达到了对氟咯菌 腈结构进行改造和优化，以期提高其生物活性的目的， 为之后更深入地研究提供了有益的数据支持.

本文合成的吡唑联吡咯类化合物还有 4 个正在相同 的条件下进行测试中, 其结果将在后续的报道中呈现.

\section{2 结论}

本文以乙酰乙酸乙酯(三氟乙酰乙酸乙酯)和甲基肼 (苯肼)为起始原料, 经环化、甲酰化、取代、缩合、再 环化等多步反应，得到了 10 个一系列未被文献报道的 新型吡唑联吡咯类化合物, 通过该合成路线获得的产品 纯度较好、收率较高, 反应条件温和, 后处理简便, 可操 作性强, 适合规模化生产. 同时研究了溶剂、反应温度、 反应时间、物料配比、催化剂等反应条件及因素对各步 
反应的影响, 确定了各步反应的最佳条件, 获得了目标 化合物及其中间体的液相色谱分析测试条件, 并且通过 挥发法培养得到了 6 个目标化合物的单晶, 采用 ${ }^{1} \mathrm{H}$ NMR, FTIR, X 射线衍射、元素分析和熔点测定等测试 手段对目标化合物及其中间体的结构进行了表征与确 认. 对其中 6 个目标化合物进行了杀菌活性的测试, 杀 菌活性实验结果表明, 在 $200 \mathrm{mg} / \mathrm{L}$ 时不同化合物显示 出不同的杀菌活性, 其中 $11 \mathrm{a}, 11 \mathrm{c}$ 对黄瓜霜霉病抑菌效 果达到甚至超过了参照物所体现出的抑菌效果, 可作为 先导化合物对其结构进行深入的研究, 为新农药杀菌剂 的开发提供了理论支持.

\section{3 实验部分}

\section{1 仪器与试剂}

MPA100 全自动熔点仪(温度计未校正); Bruker-400 型核磁共振仪 (以 $\mathrm{CDCl}_{3}$ 或 DMSO- $d_{6}$ 做溶剂, 以 TMS 为 内标); 红外光谱仪 FTIR-8400S; Yanaco MT-3CHN 元素 分析仪; 高压液相色谱仪 LC-20A. 主要原料乙酰乙酸 乙酯、甲基肼、氧基乙酰胺为工业品, 其余试剂均为化 学纯或试剂纯.

各化合物纯度均由高效液相色谱仪测试结果显示 数据为依据, 液相测试方法: 面积归一化法; 液相色谱 分析条件: $\mathrm{C}_{18}$ 反相硅胶柱, 波长 $230 \mathrm{~nm}$, 流动相为甲 醇 / 磷酸水 $(0.5 \%$ 磷酸 $)(V: V=80: 20)$, 流速 0.8 $\mathrm{mL} / \mathrm{min}$.

\section{2 实验方法}

\subsubsection{TosMIC-对甲苯磺酰甲基异腈(4)的合成}

对甲苯亚磺酸钠 $(2)$ 的合成. 在装有温度计、摚拌 器、回流冷凝管、滴液漏斗的 $3 \mathrm{~L}$ 四口瓶中加入无水亚 硫酸钠 $600 \mathrm{~g}(4.76 \mathrm{~mol}) 、$ 碳酸氢钠 $420 \mathrm{~g}(5 \mathrm{~mol}) 、 2.4 \mathrm{~L}$ 水, 升温至 70 80 ${ }^{\circ} \mathrm{C}$, 在 $3 \mathrm{~h}$ 内分批加入对甲基苯磺酰 氯 $484 \mathrm{~g}(2.54 \mathrm{~mol})$, 每次加 5 10 g, 加完后保持温度 $80{ }^{\circ} \mathrm{C}$ 继续反应 $1 \mathrm{~h}$, 停止反应, 冰水冷却, 放置 $1 \mathrm{~h}$ 后, 过滤，得 $434 \mathrm{~g}$ 白色固体 2, 纯度 99\%, 收率 $98 \%$.

对甲苯磺酰甲基甲酰胺(3)的合成. 在装有温度计、 搅拌器、回流冷凝管(并装有导气管)、滴液漏斗的 $3 \mathrm{~L}$ 四口瓶中加入 $428 \mathrm{~g}(2.33 \mathrm{~mol})$ 上一步反应得到的白色 固体 2, 多聚甲醛 $279.8 \mathrm{~g}(10.49 \mathrm{~mol})$, 甲酰胺 $700 \mathrm{~mL}$ $(790.6 \mathrm{~g}, 17.5 \mathrm{~mol})$ 和无水甲酸 $443 \mathrm{~mL}(540.4 \mathrm{~g}, 11.66$ $\mathrm{mol}$ ), 混合物加热到 $90{ }^{\circ} \mathrm{C}$, 保持此温度反应 $2 \mathrm{~h}$, 冷却 至室温, 加入 $1750 \mathrm{~mL}$ 水, 冰水冷却, 温度降到 $40{ }^{\circ} \mathrm{C}$ 时 有白色固体析出, 继续摚拌 $1.5 \mathrm{~h}$, 过滤, 滤饼用水洗涤, 干燥后, 得到 $387.7 \mathrm{~g}$ 白色固体 3, 纯度 $98 \%$, 收率 78.1\%. m.p. 109 $110{ }^{\circ} \mathrm{C} ;{ }^{1} \mathrm{H}$ NMR (400 MHz, $\left.\mathrm{CDCl}_{3}\right) \delta$ : 2.47 (s, 3H, $\left.\mathrm{CH}_{3}\right), 4.73$ (d, $\left.J=6.9 \mathrm{~Hz}, 2 \mathrm{H}, \mathrm{CH}_{2}\right), 7.41$ (dd,
$J=17.5,8.1 \mathrm{~Hz}, 2 \mathrm{H}, \mathrm{ArH}), 7.81(\mathrm{~d}, J=8.3 \mathrm{~Hz}, 2 \mathrm{H}, \mathrm{ArH})$, $8.10(\mathrm{~s}, 1 \mathrm{H}, \mathrm{CH})$.

对甲苯磺酰甲基异睛(4)的合成. 在装有温度计、摚 拌器、回流冷凝管(并装有导气管)、滴液漏斗的 $1 \mathrm{~L}$ 四 口瓶中加入中间体 $342.6 \mathrm{~g}(0.2 \mathrm{~mol})$ 、二氯甲烷 $300 \mathrm{~mL}$ 、 三乙胺 $112 \mathrm{~mL}(0.8 \mathrm{~mol})$, 用冰盐浴冷却到 $-3{ }^{\circ} \mathrm{C}$, 滴加 三氯氧磷 $20 \mathrm{~mL}(0.22 \mathrm{~mol})$ 和 $20 \mathrm{~mL}$ 二氯甲烷的混合溶 液, 并控制温度在 $0{ }^{\circ} \mathrm{C}$ 以下, $3 \mathrm{~h}$ 滴加完, 再摚拌 $1 \mathrm{~h}$, 加 入 $300 \mathrm{~mL}$ 质量浓度 7\%的氢氧化钠溶液, 搅拌 $0.5 \mathrm{~h}$ 后 分液, 有机层用水洗涤一次, 用无水硫酸钠干燥, 过滤, 滤液先常压再减压蒸馏二氯甲烷至瓶壁上有固体出现 为止, 然后向蒸馏瓶中加入 $50 \mathrm{~mL}$ 石油醚, 冰水冷却下 静置 $1 \mathrm{~h}$, 有晶体析出, 抽滤、干燥, $32.1 \mathrm{~g}$ 固体, 纯度 93.2\%, 用石油醚重结晶后, 得浅棕色固体 $428.6 \mathrm{~g}$, 纯 度 97.8\%, 收率 73.4\%. m.p. 112.7 113.2 ${ }^{\circ} \mathrm{C}$ (文献 值 $\left.{ }^{[11]} 109.0 \sim 113.0{ }^{\circ} \mathrm{C}\right) ;{ }^{1} \mathrm{H}$ NMR $\left(400 \mathrm{MHz}, \mathrm{CDCl}_{3}\right) \delta$ : $2.50\left(\mathrm{~s}, 3 \mathrm{H}, \mathrm{CH}_{3}\right), 4.58\left(\mathrm{~s}, 2 \mathrm{H}, \mathrm{CH}_{2}\right), 7.45(\mathrm{~d}, J=8.0 \mathrm{~Hz}$, $2 \mathrm{H}, \mathrm{ArH}), 7.89$ (d, $J=8.3 \mathrm{~Hz}, 2 \mathrm{H}, \mathrm{ArH}$ ).

\subsubsection{1,3-二甲基-5-吡唑酮(7a)的合成}

参考文献[12]的方法, 在装有温度计、搅拌器、滴 液漏斗和回流冷凝管的 $1 \mathrm{~L}$ 四口瓶中加入乙酰乙酸乙酯 $195 \mathrm{~g}$ (1.5 mol ), 向装有乙酰乙酸乙酯的反应瓶中滴加 $180 \mathrm{~g}(1.5 \mathrm{~mol})$ 质量浓度为 40\%的甲基肼, 通过冷水浴 控温在 $16{ }^{\circ} \mathrm{C}$ 的条件下反应 $2 \mathrm{~h}$, 采用减压蒸馏 $3 \mathrm{~h}$, 然 后向反应体系中加入甲苯常压回流分水，除水后冷却降 温析出浅黄色固体, 固体经过滤和干燥后得 $159.9 \mathrm{~g}$, 纯 度 99.85\%, 收率 95.2\%. m.p. 117.1 117.5 ${ }^{\circ} \mathrm{C}$ (文献 值 $\left.{ }^{[14]} 117.0{ }^{\circ} \mathrm{C}\right) ;{ }^{1} \mathrm{H}$ NMR (400 MHz, DMSO- $\left.d_{6}\right) \delta: 2.07$ (s, $3 \mathrm{H}, \mathrm{CH}_{3}$ ), 3.15 (s, $2 \mathrm{H}, \mathrm{CH}_{2}$ ), 3.24 (s, $3 \mathrm{H}, \mathrm{NCH}_{3}$ ).

\subsubsection{5-氯-1,3-二甲基- $1 H$-吡唑-4-甲醛 $(\mathbf{8 a})$ 的合成}

参考文献[15]的方法, 将上一步得到的 $126.7 \mathrm{~g}$ (1.13 mol) 7a 加入到 $166.7 \mathrm{~g}(2.28 \mathrm{~mol}) \mathrm{N}, \mathrm{N}$-二甲基甲酰 胺(DMF)溶液中, 在 $10{ }^{\circ} \mathrm{C}$ 的温度下滴加 $433.6 \mathrm{~g}(2.82$ $\mathrm{mol})$ 三氯氧磷, 然后升温至 $110{ }^{\circ} \mathrm{C}$, 反应 $6 \mathrm{~h}$ 后倒入冰 水中搅拌 $30 \mathrm{~min}$, 用乙酸乙酯萃取三次, 萃取液经洗涤 后用无水硫酸镁干燥, 再浓缩得到淡黄色固体 $116.5 \mathrm{~g}$, 纯度 $98.7 \%$, 收率 $64.97 \%$, m.p. $78.3 \sim 78.8{ }^{\circ} \mathrm{C}$ (文献 值 $\left.{ }^{[15]} 78 \sim 79{ }^{\circ} \mathrm{C}\right) ;{ }^{1} \mathrm{H}$ NMR $\left(400 \mathrm{MHz}, \mathrm{CDCl}_{3}\right) \delta: 2.46(\mathrm{~s}$, $\left.3 \mathrm{H}, \mathrm{CH}_{3}\right), 3.83\left(\mathrm{~s}, 3 \mathrm{H}, \mathrm{NCH}_{3}\right), 9.87(\mathrm{~s}, 1 \mathrm{H}, \mathrm{CH})$.

3.2.4 5-(4-氟苯氧基)-1,3-二甲基- $1 H$-吡唑-4-甲醛 (9a)的合成

参考文献[18]的方法, 在装有温度计, 搅拌器, 回 流冷凝管的 $250 \mathrm{~mL}$ 四口瓶中加入 $80 \mathrm{~mL}$ DMF, $6.2 \mathrm{~g}$ $(0.11 \mathrm{~mol})$ 氢氧化钾和 $11.2 \mathrm{~g}(0.1 \mathrm{~mol})$ 对氟苯酚, 加热到 $80{ }^{\circ} \mathrm{C}$, 傥拌反应 $1 \mathrm{~h}$, 然后降温到 $60{ }^{\circ} \mathrm{C}$, 再加入 $15.9 \mathrm{~g}$ 
(0.1 mol) 8a, 加热至 $90{ }^{\circ} \mathrm{C}$ 反应 $4 \mathrm{~h}$, 加入 $100 \mathrm{~mL}$ 去水继 续摚拌 $10 \mathrm{~min}$, 再用甲苯萃取三次, 萃取液用无水硫酸 镁干燥后浓缩得到浅黄色固体 $18.5 \mathrm{~g}$, 纯度 $97.8 \%$, 收 率 79.1\%. m.p. $75.0 \sim 75.6{ }^{\circ} \mathrm{C}$; ${ }^{1} \mathrm{H}$ NMR $(400 \mathrm{MHz}$, $\left.\mathrm{CDCl}_{3}\right) \delta: 2.47\left(\mathrm{~s}, 3 \mathrm{H}, \mathrm{CH}_{3}\right), 3.66\left(\mathrm{~s}, 3 \mathrm{H}, \mathrm{NCH}_{3}\right), 6.95 \sim$ $7.11(\mathrm{~m}, 4 \mathrm{H}, \mathrm{ArH}), 9.54(\mathrm{~s}, 1 \mathrm{H}, \mathrm{CH})$.

3.2.5 2-氰基-3-(5-(4-氟苯氧基)-1,3-二甲基- $1 H$-吡 唑-4-基)丙烯酰胺 (10a) 的合成

参考文献[20]的方法, 在装有温度计, 搅拌器, 回 流冷凝管的 $250 \mathrm{~mL}$ 四口瓶中装入 $80 \mathrm{~mL}$ 乙醇, 加入 $11.7 \mathrm{~g}(0.05 \mathrm{~mol}) 9 \mathrm{~b} 、 5 \mathrm{~g}(0.06 \mathrm{~mol})$ 诵基乙酰胺和 $6.6 \mathrm{~g}$ $(0.06 \mathrm{~mol})$ 三乙胺, 加热回流 $2 \mathrm{~h}$, 然后加入 $100 \mathrm{~mL}$ 水摚 拌 $20 \mathrm{~min}$, 析出过滤得到浅黄色固体 $10 \mathrm{~g}$, 纯度 $97.8 \%$, 收率 66.7\%. m.p. $218.3 \sim 218.5{ }^{\circ} \mathrm{C} ;{ }^{1} \mathrm{H}$ NMR $(400 \mathrm{MHz}$, $\left.\mathrm{CDCl}_{3}\right) \delta: 2.29\left(\mathrm{~s}, 3 \mathrm{H}, \mathrm{CH}_{3}\right), 3.58\left(\mathrm{~s}, 3 \mathrm{H}, \mathrm{NCH}_{3}\right), 6.92 \sim$ $7.02(\mathrm{~m}, 4 \mathrm{H}, \mathrm{ArH}), 7.17 \sim 7.28(\mathrm{~m}, 4 \mathrm{H}, \mathrm{ArH}), 7.61$ (d, $J=$ $\left.33.8 \mathrm{~Hz}, 2 \mathrm{H}, \mathrm{NH}_{2}\right), 7.76$ (s, $\left.1 \mathrm{H}, \mathrm{CH}\right)$.

\subsection{6 目标化合物 $11 \mathrm{a} \sim 11 \mathrm{j}$ 的合成通法}

参考文献[5]的方法, 在装有温度计、搅拌器、回流 冷凝管、滴液漏斗的 $250 \mathrm{~mL}$ 的四口瓶中装入 $50 \mathrm{~mL}$ 甲 醇, 加入 $0.01 \mathrm{~mol}$ 化合物 10 和 $1.95 \mathrm{~g}(0.01 \mathrm{~mol}) \mathbf{4}$, 在冰 浴的条件下滴加由 $20 \mathrm{~mL}$ 甲醇和 $1.12 \mathrm{~g}(0.02 \mathrm{~mol}) \mathrm{KOH}$ 组成的混合液, 在室温下反应 $3 \mathrm{~h}$ 后减压蒸出甲醇, 加 入 $50 \mathrm{~mL}$ 水搅拌 $0.5 \mathrm{~h}$, 过滤干燥后用甲醇重结晶得到目 标化合物 $\mathbf{1 1 a} \sim \mathbf{1 1}$ j.

4-[1,3-二甲基-5-(4-氟苯氧基)- $1 H$-吡唑-4-基]- $1 H$-吡 咯-3-腈(11a): 白色固体, 纯度 $98.1 \%$, 收率 $87.8 \%$. 液相 分析产物保留时间 4.528 min. m.p. $205.6 \sim 206.3{ }^{\circ} \mathrm{C} ;{ }^{1} \mathrm{H}$ NMR (400 MHz, DMSO- $\left.d_{6}\right) \delta: 2.15\left(\mathrm{~s}, 3 \mathrm{H}, \mathrm{CH}_{3}\right), 3.57$ (s, $\left.3 \mathrm{H}, \mathrm{NCH}_{3}\right), 6.66(\mathrm{~s}, 1 \mathrm{H}$, Pyrrole-5-CH), $6.80 \sim 6.99(\mathrm{~m}$, 2H, ArH), 7.12 (t, $J=8.7 \mathrm{~Hz}, 2 \mathrm{H}, \mathrm{ArH}), 7.52$ (s, 1H, Pyrrole-2-CH), 11.67 (brs, 1H, NH); IR (KBr) v: 3386, 3148, $3076,2948,2216,1683,832,769 \mathrm{~cm}^{-1}$. Anal. calcd for $\mathrm{C}_{16} \mathrm{H}_{13} \mathrm{~N}_{4}$ : C 64.83, H 4.44, N 18.89; found C 64.86, H 4.42, N 18.91 .

4-[1,3-二甲基-5-(4-甲苯氧基)- $1 H$-吡唑-4-基]- $1 H$-吡 咯-3-腈(11b): 白色晶状固体, 纯度 98.9\%, 收率 75.9\%。 液相分析产物保留时间 4.968 min. m.p. 224.4 224.6 ${ }^{\circ} \mathrm{C} ;{ }^{1} \mathrm{H}$ NMR (400 MHz, $\left.\mathrm{CDCl}_{3}\right) \delta: 2.26\left(\mathrm{~s}, 3 \mathrm{H}, \mathrm{CH}_{3}\right), 2.32$ (s, $\left.3 \mathrm{H}, \mathrm{CH}_{3}\right), 3.63\left(\mathrm{~s}, 3 \mathrm{H}, \mathrm{NCH}_{3}\right), 6.55$ (t, $J=2.3 \mathrm{~Hz}, 1 \mathrm{H}$, Pyrrole-5-CH), 6.77 (d, $J=8.6 \mathrm{~Hz}, 2 \mathrm{H}, \mathrm{ArH}), 7.03$ (d, $J=$ $8.4 \mathrm{~Hz}, 2 \mathrm{H}, \mathrm{ArH}), 7.18 \sim 7.25$ (m, 1H, Pyrrole-2-CH), 8.64 (brs, 1H, NH); IR (KBr) v: 3445, 3127, 3075, 2946, 2215, $1607,811 \mathrm{~cm}^{-1}$. Anal. calcd for $\mathrm{C}_{17} \mathrm{H}_{16} \mathrm{~N}_{4}$ : C $69.88, \mathrm{H}$ 5.50, N 19.16; found C 69.85, H 5.52, N 19.17.
4-[5-(4-叔丁基苯氧基)-1,3-二甲基- $1 \mathrm{H}$-吡唑-4基]-1 $H$-吡咯-3-腈(11c): 白色晶状固体, 纯度 $96.9 \%$, 收 率 $68.0 \%$. 液相分析产物保留时间 6.881 min. m.p. $202.1 \sim 202.4{ }^{\circ} \mathrm{C} ;{ }^{1} \mathrm{H}$ NMR (400 MHz, $\left.\mathrm{CDCl}_{3}\right) \delta: 1.25$ (s, $\left.9 \mathrm{H},\left(\mathrm{CH}_{3}\right)_{3}\right), 2.31\left(\mathrm{~s}, 3 \mathrm{H}, \mathrm{CH}_{3}\right), 3.61\left(\mathrm{~s}, 3 \mathrm{H}, \mathrm{NCH}_{3}\right), 6.55$ (t, $J=2.3 \mathrm{~Hz}, 1 \mathrm{H}$, Pyrrole-5-CH), 6.79 (d, $J=8.8 \mathrm{~Hz}, 2 \mathrm{H}$, $\mathrm{ArH}), 7.19 \sim 7.21(\mathrm{~m}, 1 \mathrm{H}$, Pyrrole-2-CH), 7.23 (d, $J=8.8$ $\mathrm{Hz}, 2 \mathrm{H}, \mathrm{ArH}), 8.94$ (brs, 1H, NH); IR (KBr) v: 3536, 3139, 2964, 2223, 1504, 1221, $823 \mathrm{~cm}^{-1}$. Anal. calcd for $\mathrm{C}_{20} \mathrm{H}_{22} \mathrm{~N}_{4}$ : C 71.77, H 6.66, N 16.77; found C 71.83, H 6.63, N 16.75 .

4-[5-(4-甲氧基苯氧基)-1,3-二甲基- $1 H$ - 吡唑-4基]-1H-吡咯-3-腈(11d): 粉色晶状固体, 纯度 97.7\%, 收 率 $56.3 \%$. 液相分析产物保留时间 $4.522 \mathrm{~min}$. m.p. $202.0 \sim 203.0{ }^{\circ} \mathrm{C} ;{ }^{1} \mathrm{H}$ NMR (400 MHz, DMSO- $\left.d_{6}\right) \delta: 2.15$ (s, $3 \mathrm{H}, \mathrm{CH}_{3}$ ), 3.55 (s, $\left.3 \mathrm{H}, \mathrm{NCH}_{3}\right), 3.68$ (s, $\left.3 \mathrm{H}, \mathrm{OCH}_{3}\right), 6.65$ (s, 1H, Pyrrole-5-CH), 6.82 (td, J=9.3, 2.6 Hz, 4H, ArH), 7.53 (s, 1H, Pyrrole-2-CH), 11.67 (brs, 1H, NH); IR (KBr) $v: 3386,3136,2946,2908,2218,1496,1190,826,799$ $\mathrm{cm}^{-1}$. Anal. calcd for $\mathrm{C}_{17} \mathrm{H}_{16} \mathrm{~N}_{4}$ : C 66.24, H 5.25, N 18.12; found $\mathrm{C} 66.22, \mathrm{H} 5.23, \mathrm{~N} 18.17$.

4-[5- 苯氧基-1-甲基-3-(三氟甲基)- $1 \mathrm{H}$ - 吡唑-4基]-1 $H$-吡咯-3-腈(11e): 白色晶状固体, 纯度 $98.8 \%$, 收 率 $66.1 \%$. 液相分析产物保留时间 4.806 min. m.p. $179.7 \sim 180.3{ }^{\circ} \mathrm{C} ;{ }^{1} \mathrm{H}$ NMR (400 MHz, DMSO- $\left.d_{6}\right) \delta: 3.76$ (s, 3H, $\left.\mathrm{NCH}_{3}\right), 6.76(\mathrm{t}, J=2.3 \mathrm{~Hz}, 1 \mathrm{H}$, Pyrrole-5-CH), 6.92 (dd, $J=8.7,0.9 \mathrm{~Hz}, 2 \mathrm{H}, \mathrm{ArH}), 7.08(\mathrm{t}, J=7.4 \mathrm{~Hz}, 1 \mathrm{H})$, $7.26 \sim 7.35(\mathrm{~m}, 2 \mathrm{H}, \mathrm{ArH}), 7.53(\mathrm{dd}, J=3.1,2.0 \mathrm{~Hz}, 1 \mathrm{H}$, Pyrrole-2-CH), 11.77 (brs, 1H, NH); IR (KBr) v: 3218, $3124,2232,1594,1485,1159,754 \mathrm{~cm}^{-1}$. Anal. calcd for $\mathrm{C}_{16} \mathrm{H}_{11} \mathrm{~N}_{4}$ : C 57.82, H 3.30, N 16.90; found C 57.83, H 3.34, N 16.86 .

4-[5-(4-氟苯氧基)-1-甲基-3-(三氟甲基)- $1 H$-吡唑-4基]-1H-吡咯-3-腈(11f): 浅黄色晶状固体，纯度 97.8\%, 收率 91.4\%. 液相分析产物保留时间 4.564 min. m.p. $138.7 \sim 139.1{ }^{\circ} \mathrm{C} ;{ }^{1} \mathrm{H}$ NMR (400 MHz, DMSO-d $\left.d_{6}\right) \delta: 3.79$ (s, 3H, $\left.\mathrm{NCH}_{3}\right), 6.75(\mathrm{~s}, 1 \mathrm{H}$, Pyrrole-5-CH), 6.94 7.02 (m, $2 \mathrm{H}, \operatorname{ArH}), 7.07 \sim 7.17(\mathrm{~m}, 2 \mathrm{H}, \operatorname{ArH}), 7.52$ (s, 1H, Pyrrole-2-CH), 11.76 (brs, 1H, NH); IR (KBr) v: 3225, 3122, 2905, 2231, 1609, 1498, 1164, 831, $772 \mathrm{~cm}^{-1}$. Anal. calcd for $\mathrm{C}_{16} \mathrm{H}_{10} \mathrm{~N}_{4}$ : C 54.88, H 2.87, N 16.01; found C 54.86, H 2.88, N 16.00 .

4-[5-(4-甲苯氧基)-1-甲基-3-(三氟甲基)-1 $H$-吡唑-4基]-1H-吡咯-3-腈(11g)：白色晶状固体，纯度 99.2\%, 收 率 $53.4 \%$. 液相分析产物保留时间 7.603 min. m.p. 
193.4 194.1 ${ }^{\circ} \mathrm{C} ;{ }^{1} \mathrm{H}$ NMR (400 MHz, DMSO- $\left.d_{6}\right) \delta: 2.21$ (s, 3H, $\left.\mathrm{CH}_{3}\right), 3.74\left(\mathrm{~s}, 3 \mathrm{H}, \mathrm{NCH}_{3}\right), 6.75(\mathrm{t}, J=1.9 \mathrm{~Hz}, 1 \mathrm{H}$, Pyrrole-5-CH), 6.80 (d, $J=8.6 \mathrm{~Hz}, 2 \mathrm{H}, \mathrm{ArH}), 7.08$ (m, $J=$ $8.2 \mathrm{~Hz}, 2 \mathrm{H}, \mathrm{ArH}), 7.52$ (d, $J=1.9 \mathrm{~Hz}, 1 \mathrm{H}$, Pyrrole-2-CH), 11.74 (brs, 1H, NH); IR (KBr) v: 3191, 2910, 2226, 1620, 1504, 1165, 820, $777 \mathrm{~cm}^{-1}$. Anal. calcd for $\mathrm{C}_{17} \mathrm{H}_{13} \mathrm{~N}_{4}$ : C 59.01, H 3.77, N 16.13; found C 58.96, H 3.78, N 16.18.

4-[5-(4-(叔丁基)苯氧基)-1-甲基-3-(三氟甲基)- $1 H$ 吡唑-4-基]- $1 H$-吡咯-3-腈(11h)：浅色晶状固体，纯度 99.4\%, 收率 75.6\%. 液相分析产物保留时间 $7.686 \mathrm{~min}$. m.p. $179.1 \sim 180.0{ }^{\circ} \mathrm{C} ;{ }^{1} \mathrm{H}$ NMR $\left(400 \mathrm{MHz}, \mathrm{DMSO}-d_{6}\right) \delta$ : $2.08\left(\mathrm{~s}, 9 \mathrm{H},\left(\mathrm{CH}_{3}\right)_{3}\right), 3.74\left(\mathrm{~s}, 3 \mathrm{H}, \mathrm{NCH}_{3}\right), 6.76(\mathrm{~s}, 1 \mathrm{H}, \mathrm{Pyr}-$ role-5-CH), $6.78 \sim 6.89(\mathrm{~m}, 2 \mathrm{H}, \mathrm{ArH}), 7.23 \sim 7.37(\mathrm{~m}, 2 \mathrm{H}$, ArH), 7.53 (t, $J=2.3 \mathrm{~Hz}, 1 \mathrm{H}$, Pyrrole-2-CH), 11.76 (brs, $1 \mathrm{H}, \mathrm{NH}$ ); IR (KBr) v: 3252, 2965, 2233, 1613, 1506, 1168, $767 \mathrm{~cm}^{-1}$. Anal. calcd for $\mathrm{C}_{20} \mathrm{H}_{19} \mathrm{~N}_{4}: \mathrm{C}$ 61.84, $\mathrm{H} 4.94, \mathrm{~N}$ 14.43; found C 61.85, H 4.93, N 14.43.

4-[5-(4-甲氧基苯氧基)-1-甲基-3-(三氟甲基)- $1 H$-吡 唑-4-基]-1H-吡咯-3-腈(11i)：白色晶状固体，纯度 $99.4 \%$, 收率 $68.1 \%$. 液相分析产物保留时间 $4.714 \mathrm{~min}$. m.p. $172.7 \sim 173.2{ }^{\circ} \mathrm{C} ;{ }^{1} \mathrm{H}$ NMR $\left(400 \mathrm{MHz}, \mathrm{DMSO}-d_{6}\right) \delta$ : $3.67\left(\mathrm{~s}, 3 \mathrm{H}, \mathrm{OCH}_{3}\right), 3.75\left(\mathrm{~s}, 3 \mathrm{H}, \mathrm{CH}_{3}\right), 6.73$ (d, $J=1.9 \mathrm{~Hz}$, 1H, Pyrrole-5-CH), $6.77 \sim 6.89(\mathrm{~m}, 4 \mathrm{H}, \mathrm{ArH}), 7.52(\mathrm{~d}, J=$ 1.9 Hz, 1H, Pyrrole-2-CH), 11.75 (brs, 1H, NH); IR (KBr) $v: 3393,3024,2978,2225,1618,1504,1177,892,778$ $\mathrm{cm}^{-1}$. Anal. calcd for $\mathrm{C}_{17} \mathrm{H}_{13} \mathrm{~N}_{4}$ : C 56.35, H 3.66, N 15.43; found C 56.36, H 3.62, N 15.46.

4-[5-(4- 甲苯氧基)-1- 苯基-3-甲基- $1 H$-吡唑-4基]-1H-吡咯-3-腈(11j): 黄色晶状固体, 纯度 99.2\%, 收 率 $62.5 \%$. 液相分析产物保留时间 7.741 min. m.p. 196.7 197.4 ${ }^{\circ} \mathrm{C} ;{ }^{1} \mathrm{H}$ NMR (400 MHz, DMSO- $d_{6}$ ) $\delta: 2.16$ $\left(\mathrm{s}, 3 \mathrm{H}, \mathrm{CH}_{3}\right), 2.28\left(\mathrm{~s}, 3 \mathrm{H}, \mathrm{CH}_{3}\right), 6.73(\mathrm{~s}, 1 \mathrm{H}$, Pyrrole-5-CH), 6.76 (d, $J=7.3 \mathrm{~Hz}, 2 \mathrm{H}, \mathrm{ArH}), 7.02$ (m, $J=8.2$ $\mathrm{Hz}, 2 \mathrm{H}, \mathrm{ArH}), 7.28$ (t, $J=7.2 \mathrm{~Hz}, 1 \mathrm{H}, \mathrm{ArH}), 7.43$ (t, $J=$ $7.7 \mathrm{~Hz}, 2 \mathrm{H}, \mathrm{ArH}), 7.56$ (s, 1H, Pyrrole-2-CH), 7.61 (d, $J=$ $7.9 \mathrm{~Hz}, 2 \mathrm{H}, \mathrm{ArH}), 11.74$ (br.s, 1H, NH); IR (KBr) v: 3126 , $2905,2223,1599,1502,1205,812,766 \mathrm{~cm}^{-1}$. Anal. calcd for $\mathrm{C}_{22} \mathrm{H}_{18} \mathrm{~N}_{4}$ : C 74.50, H 5.16, N 15.83; found C 74.56, H 5.12, N 15.81 .

辅助材料(Supporting information) 所合成的目标化 合物的红外、核磁共振氢谱及单分子结构图. 这些材 料可以免费从本刊网站(http://sioc-journal.cn/)上下载.

\section{References}

[1] Xu, S.-C.; Jiang, M.-G. Chin. J. Pestic. Sci. 2002, 4(2), 1 (in Chinese). (徐尚成, 蒋木庚, 农药学学报, 2002, 4(2), 1.)

[2] Gordon, S. F.; Linda, V.; Hall, J. C. J. Agric. Food Chem. 2006, 54, 688.

[3] Han, F.-G.; Lu, Y.; Ji, X.-M.; Zhao, M.-Q. Zhang, X.-Y.; Liu, Y. Chin. J. Org. Chem. 2010, 30, 1080 (in Chinese).

(韩富根, 卢叶, 姬小明, 赵铭钦, 张晓蕴, 刘云, 有机化学, 2010, 30, 1080.)

[4] Schirra, M.; Cabras, P.; Angioni, A.; D'hallewin, G.; Ruggiu, R.; Elizabeth, V. M. J. Agric. Food Chem. 1997, 45, 4127.

[5] Li, C.; Liao, D.-H.; Zeng, Z.-W, Wang, M.-J.; Wu, Z.-X.; Yang, F.; Shi, W.-J. Modern Agrochem. 2009, 8, 19 (in Chinese).

(李超，廖道华，曾仲武，王美娟，吴忠信，杨芳，师文娟，现代 农药, 2009, 8, 19.)

[6] Meng, F.-W. M. S. Thesis, Heilongjiang University, 2006 (in Chinese). (孟凡伟, 硕士论文，黑龙江大学, 2006.)

[7] Akkarapalli, M.; Guda, M. R.; Gopala, L.; Venkatapuram, P.; Adivireddy, P. J. Heterocycl. Chem. 2014, 51, 179.

[8] Hu, L.-M.; Li, X.-S.; Chen, Z.-Y.; Liu, Z.-J. Chin. J. Org. Chem. 2003, 23, 1131 (in Chinese) (胡利明, 李学怒, 陈致远, 刘钊杰, 有机化学, 2003, 23, 1131.)

[9] Sun, N.-B.; Tong, J.-Y.; Wu, H.-K. Chin. J. Org. Chem. 2013, 33, 101 (in Chinese)

(孙娜波，童建颖，武宏科，有机化学， 2013, 33, 101.)

[10] Dai, H.; Yu, H.-B.; Liu, J.-B.; Qin, X.; Wang, T.-T.; Zhang, X.; Qin, Z.-F.; Fang, J.-X. Chin. J. Org. Chem. 2013, 33, 1104 (in Chinese.)

(戴红，于海波，刘建兵，秦雪，王婷婷，张欣，秦振芳，方建新, 有机化学, 2013, 33, 1104.)

[11] Leusen, A. M. V.; Wildeman, J.; Oldenziel, O. H. J. Org. Chem. 1977, 42, 1153.

[12] Nayak, M.; Batchu, H.; Batra, S. Tetrahedron Lett. 2012, 53, 4206.

[13] Markovic, V.; Eric, S.; Juranic, Z. D.; Stanojkovic, T.; Ljubinka, J. L.; Rankovic, B.; Kosanic, M.; Joksovic, M. D. Bioorg. Chem. 2011, 39, 18

[14] Huang, Y.-Y.; Lin, H.-C.; Cheng, K.-M.; Su, W.-N.; Sung, K. C.; Lin, T.-S.; Huang, J.-J.; Lin, S.-K.; Wong, F.-F. Tetrahedron 2009, $65,9592$.

[15] Tang, X.-Y.; Shi, M. J. Org. Chem. 2008, 73, 8317.

[16] Phillips, B. A.; Fodor, G.; Gal, J.; Letourneau, F.; Ryan, J. J. Tetrahedron 1973, 29, 3309

[17] Hesse, S.; Kirsch, G. Tetrahedron Lett. 2002, 43, 1213.

[18] Qian, X.-Q.; Zhou, H.; Zhan, X.-P.; Liu, Z.-L.; Mao, Z.-M. Chin. J. Org. Chem. 2012, 32, 2223 (in Chinese).

(钱晓庆，周恒，詹晓平，刘增璐，毛振民，有机化学，2012，32， 2223.)

[19] Park, H.-J.; Lee, K.; Park, S.-J.; Ahn, B.; Lee, J.-C.; Cho H.-Y.; Lee, K.-I. Bioorg. Med. Chem. Lett. 2005, 15, 3307.

[20] Li, Y.; Zhang, H. Q.; Liu, J.; Yang, X.-P.; Liu, Z.-J. J. Agric. Food Chem. 2006, 54, 3636.

[21] Bian, Y.-J.; Qin, Y.; Xiao, L.-W.; Li, J.-T. Chin. J. Org. Chem. 2006, 26, 1165 (in Chinese) (边延江, 秦英, 肖立伟, 李记太, 有机化学, 2006, 26, 1165.)

[22] Chen, Q.-Q.; Huggins, M.-T.; Lightner, D.-A.; Norona, W.; McDonagh, A.-F. J. Am. Chem. Soc. 1999, 121, 9253. 


\title{
辅助材料(Supporting Information)
}

\section{新型吡唑联吡咯类杂环化合物的合成及杀菌活性}

\author{
王子时 ${ }^{a}$ 叶汉韬 ${ }^{a}$ 肖 丹 ${ }^{a}$ 马东升 $* a$ 高金胜 ${ }^{a, b}$ 于颖慧 $^{a}$ \\ 侯广峰*, 间鹏飞a \\ ( ${ }^{a}$ 黑龙江大学化学化工与材料学院 哈尔滨 150080) \\ ( ${ }^{b}$ 黑龙江大学农药工程中心 哈尔滨 150080)
}

\section{1 红外数据}

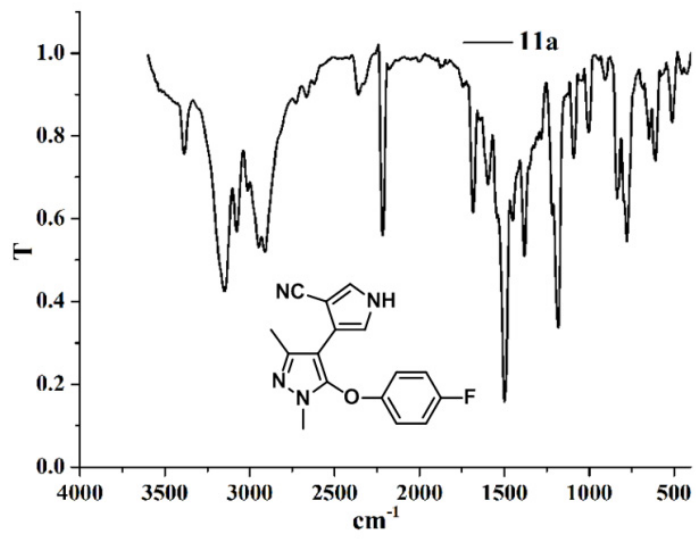

图 S1 11a 红外谱图

Figure S1 The IR spectrum of 11a

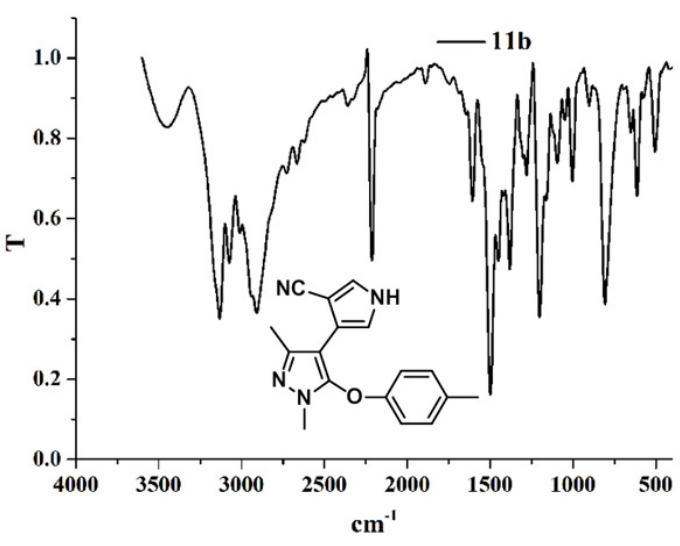

图 S2 11b 红外谱图

Figure S1 The IR spectrum of 11b

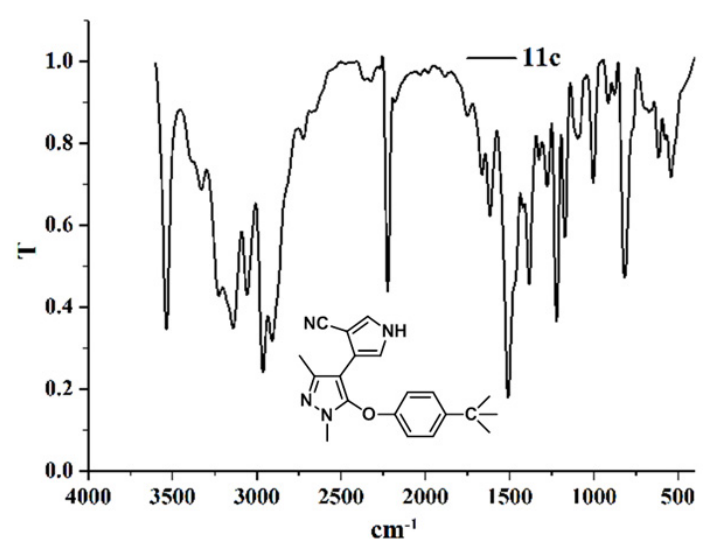

图 S3 11c 红外谱图

Figure S3 The IR spectrum of 11c

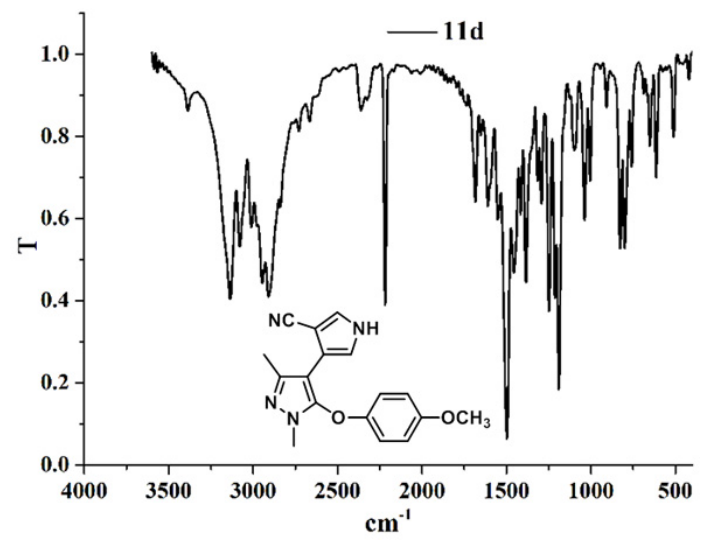

图 S4 11d 红外谱图

Figure S4 The IR spectrum of 11d 


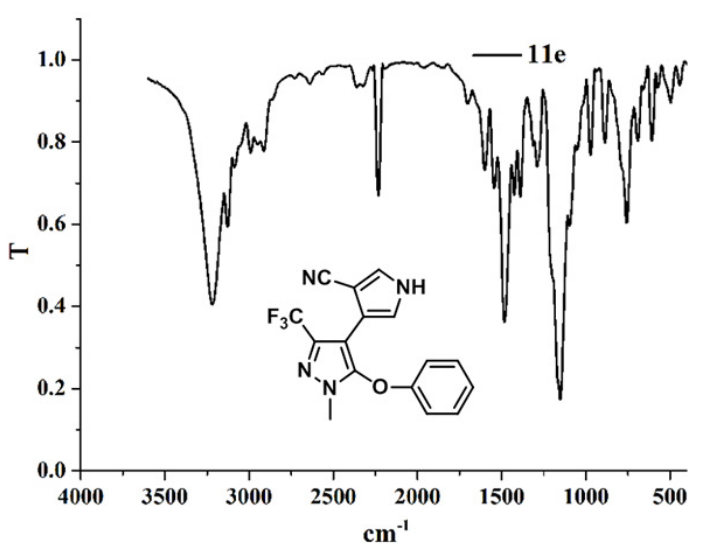

图 S5 11e 红外谱图

Figure S5 The IR spectrum of 11e

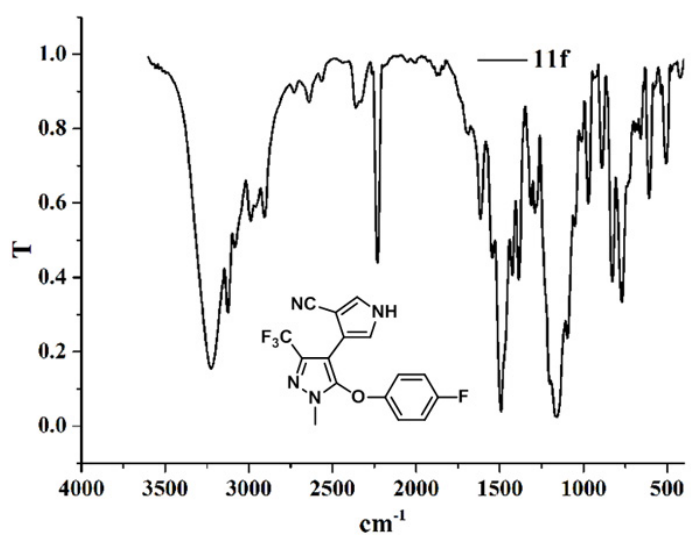

图 S6 11f 红外谱图

Figure S6 The IR spectrum of $11 f$

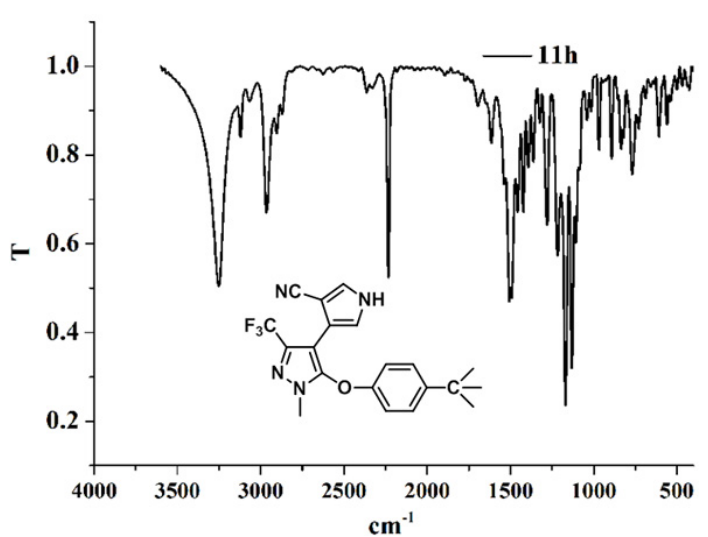

图 S8 11h 红外谱图

Figure S8 The IR spectrum of $\mathbf{1 1 h}$

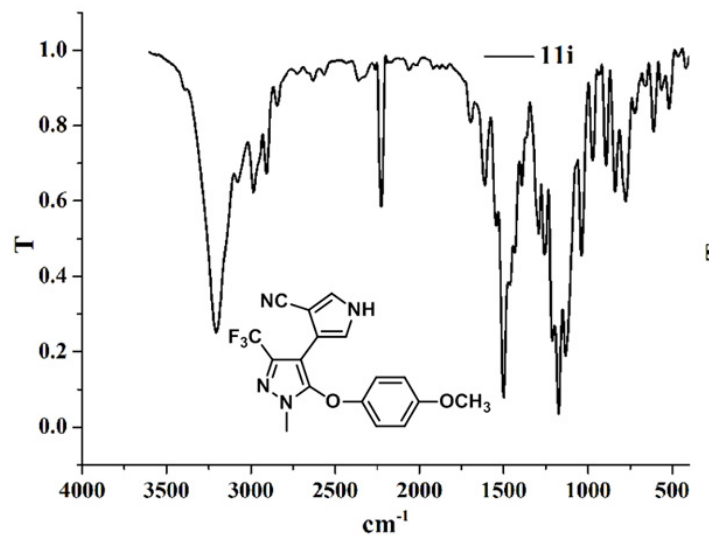

图 S9 11i 红外谱图

Figure S9 The IR spectrum of 11i

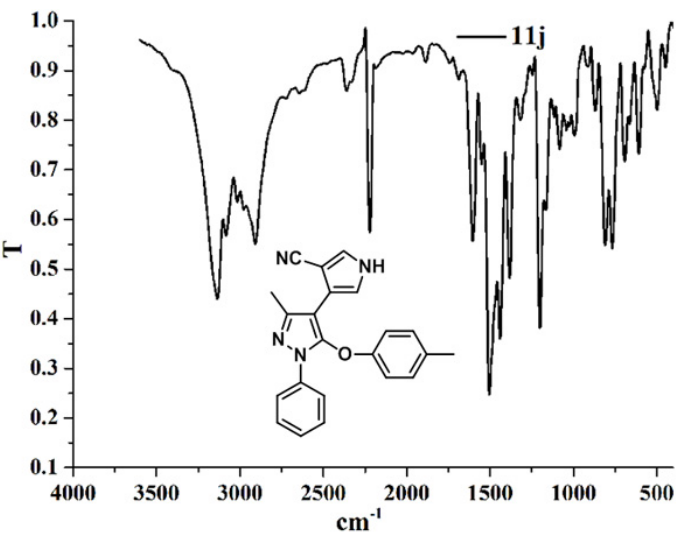

图 $\mathrm{S10} \mathrm{11j}$ 红外谱图

Figure S10 The IR spectrum of $\mathbf{1 1 j}$ 


\section{2 核磁共振氢谱}

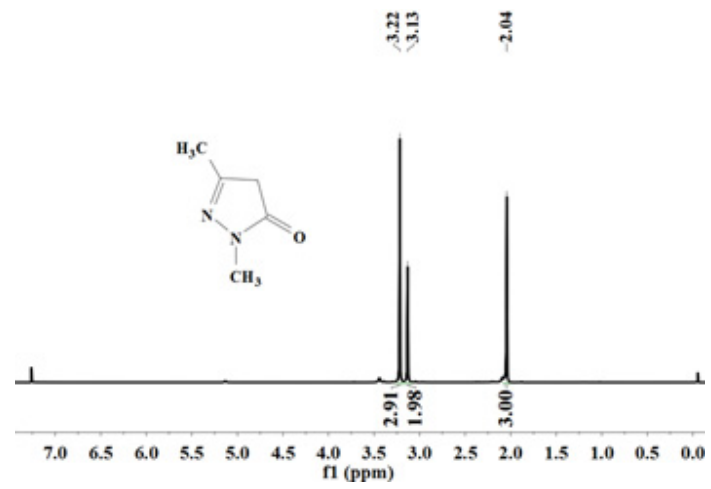

图 S11 7a 核磁共振氢谱图

Figure S11 The ${ }^{1} \mathrm{H}$ NMR spectrum of $7 \mathbf{a}$
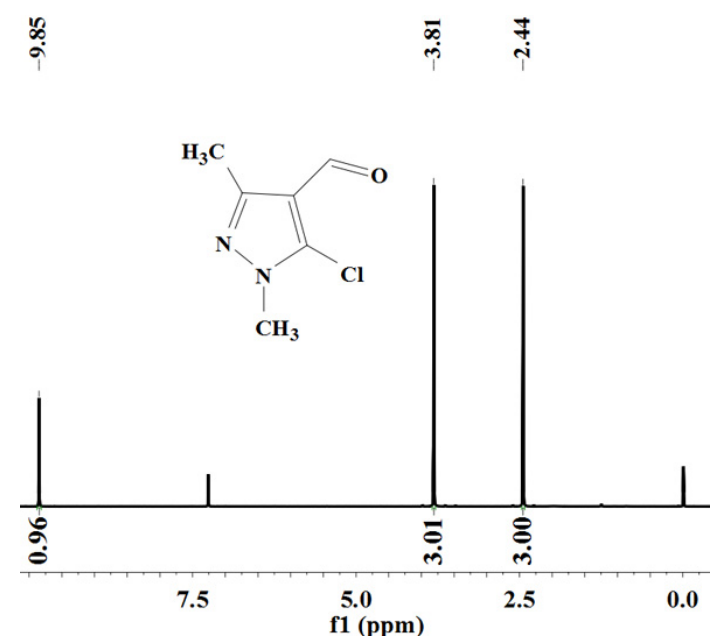

图 S12 8a 核磁共振氢谱图

Figure S12 The ${ }^{1} \mathrm{H}$ NMR spectrum of $\mathbf{8 a}$

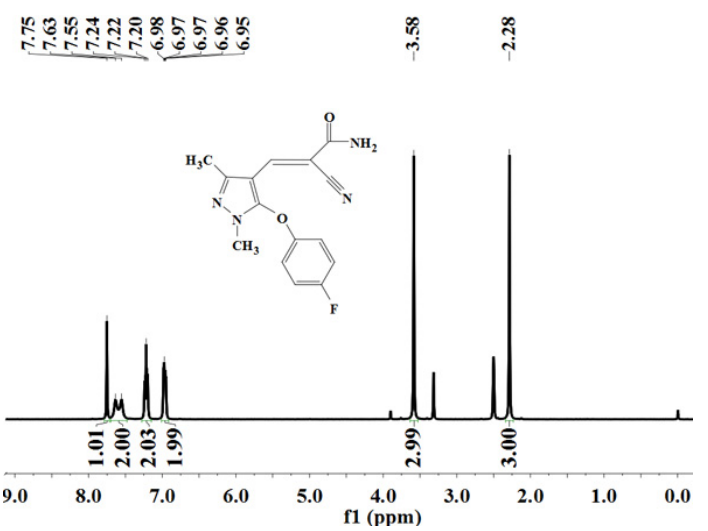

图 S14 10a 核磁共振氢谱图

Figure S14 The ${ }^{1} \mathrm{H}$ NMR spectrum of 10a

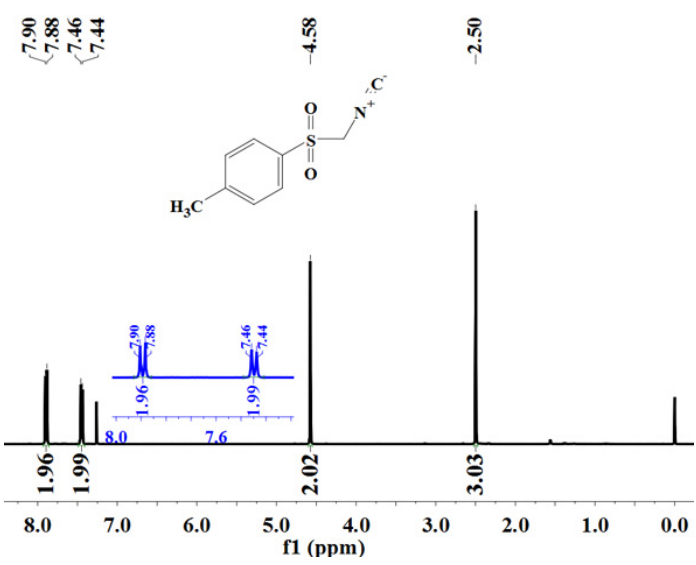

图 S16 TosMIC 核磁共振氢谱图

图 S15 化合物 3 核磁共振氢谱图

Figure S15 The ${ }^{1} \mathrm{H}$ NMR spectrum of compound 3 Figure S16 The ${ }^{1} \mathrm{H}$ NMR spectrum of TosMIC 


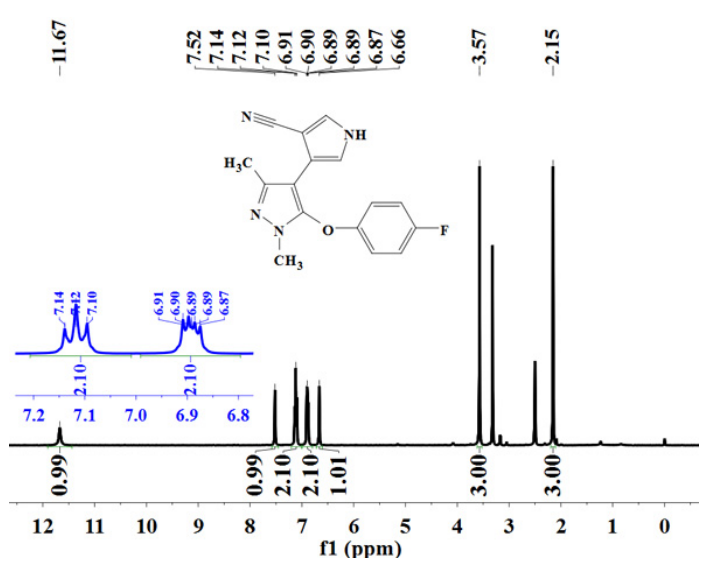

图 S17 11a 核磁共振氢谱图

Figure S17 The ${ }^{1} \mathrm{H}$ NMR spectrum of 11a
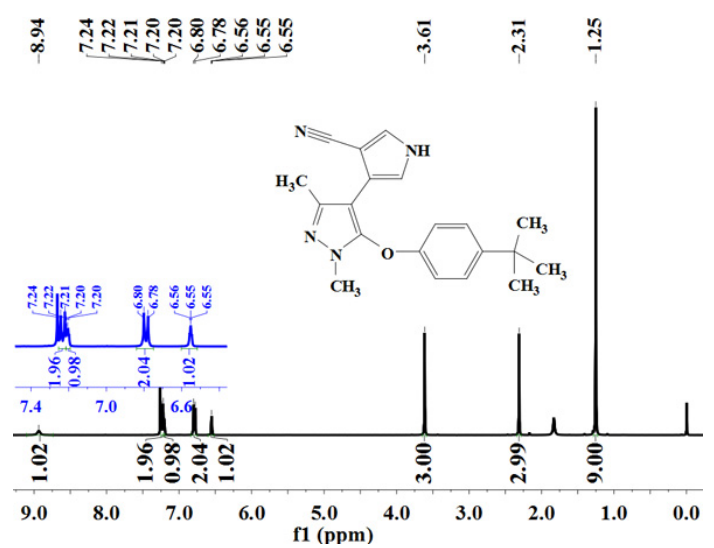

图 S19 11c 核磁共振氢谱图

Figure S19 The ${ }^{1} \mathrm{H}$ NMR spectrum of 11c

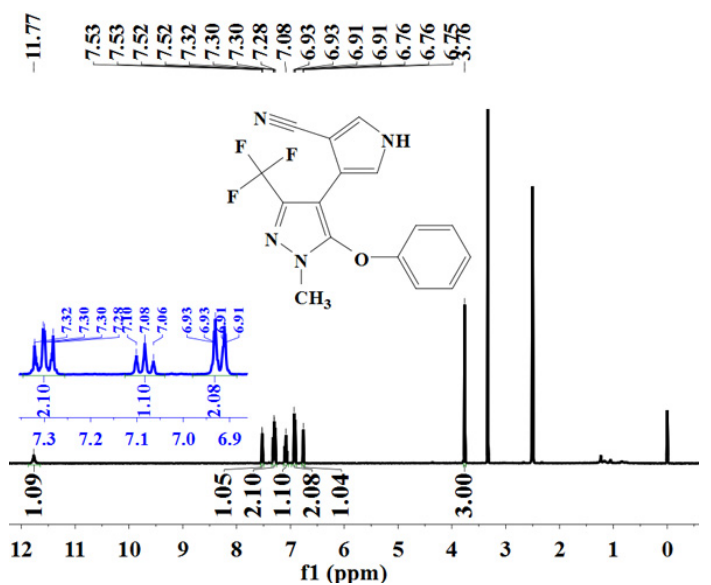

图 S21 11e 核磁共振氢谱图

Figure S21 The ${ }^{1} \mathrm{H}$ NMR spectrum of 11e

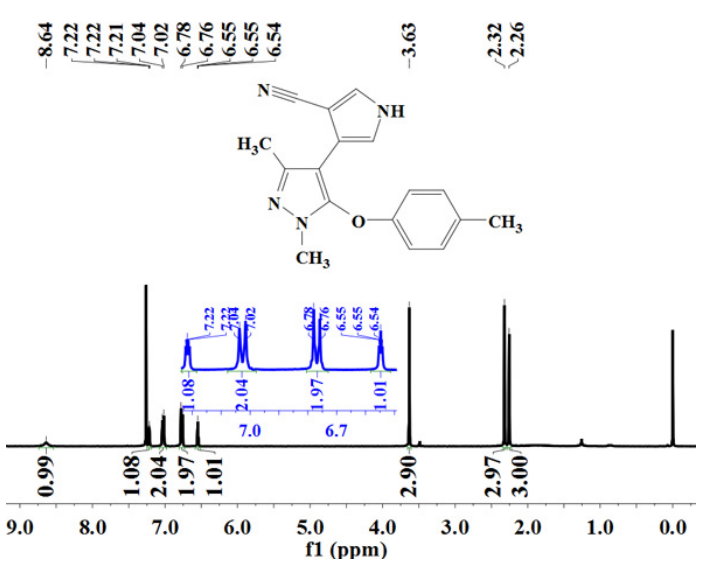

图 S18 11b 核磁共振氢谱图

Figure S18 The ${ }^{1} \mathrm{H}$ NMR spectrum of $\mathbf{1 1 b}$

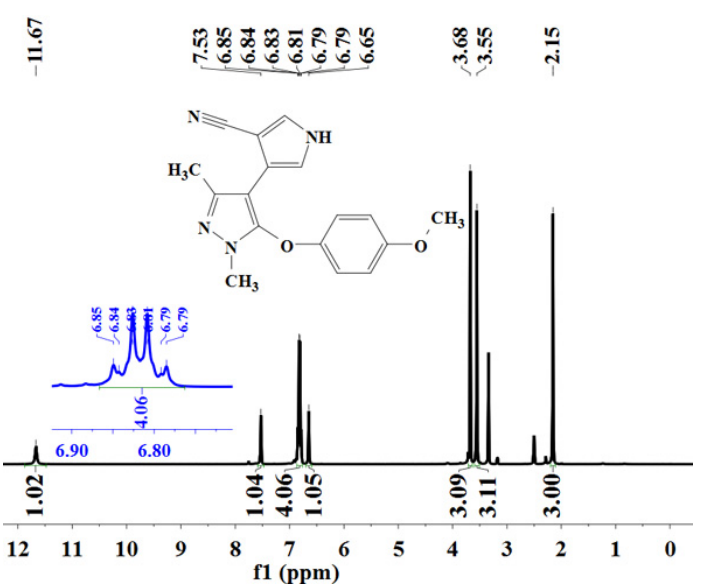

图 S20 11d 核磁共振氢谱图

Figure S20 The ${ }^{1} \mathrm{H}$ NMR spectrum of 11d

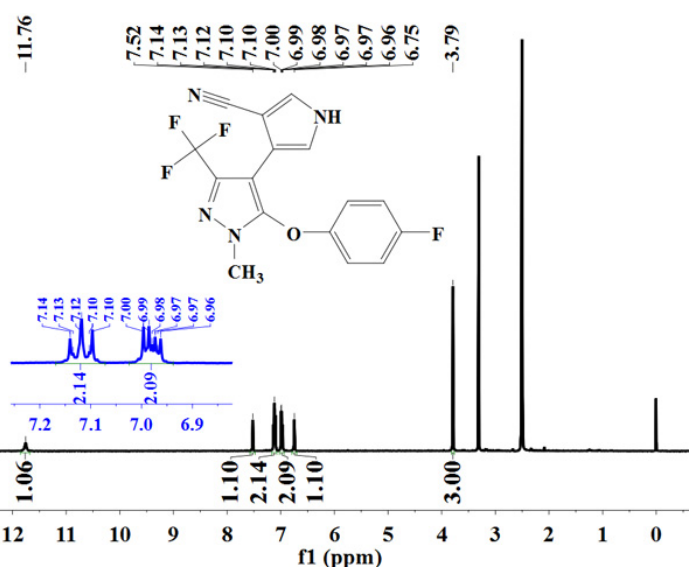

图 S22 11f 核磁共振氢谱图

Figure S22 The ${ }^{1} \mathrm{H}$ NMR spectrum of $\mathbf{1 1 f}$ 


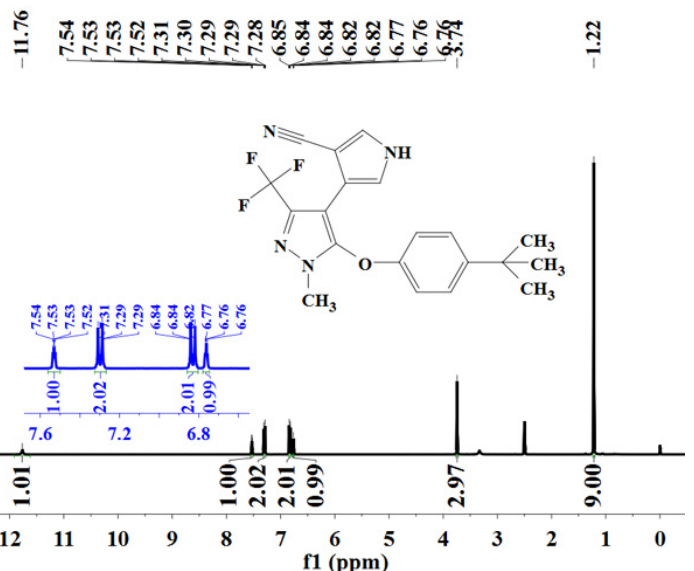

图 S23 11g 核磁共振氢谱图

Figure S23 The ${ }^{1} \mathrm{H}$ NMR spectrum of $\mathbf{1 1 g}$

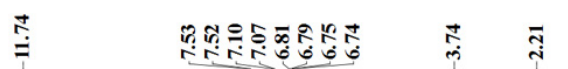
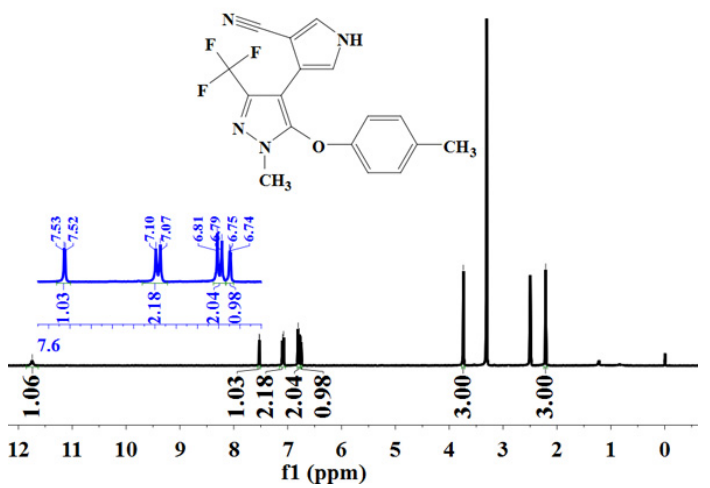

图 S24 11h 核磁共振氢谱图

Figure S24 The ${ }^{1}$ H NMR spectrum of $\mathbf{1 1 h}$

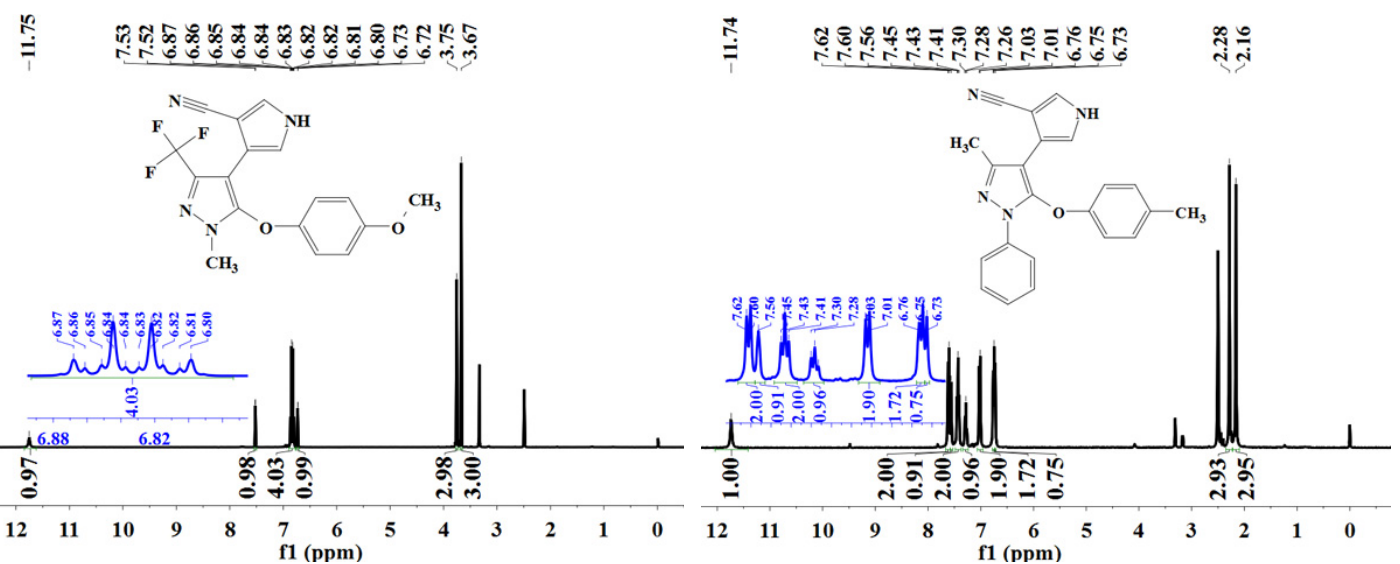

图 S25 11i 核磁共振氢谱图

Figure S25 The ${ }^{1} \mathrm{H}$ NMR spectrum of $\mathbf{1 1 i}$
图 S26 11j 核磁共振氢谱图

Figure S26 The ${ }^{1} \mathrm{H}$ NMR spectrum of $\mathbf{1 1} \mathbf{j}$

\section{3 单分子结构图}

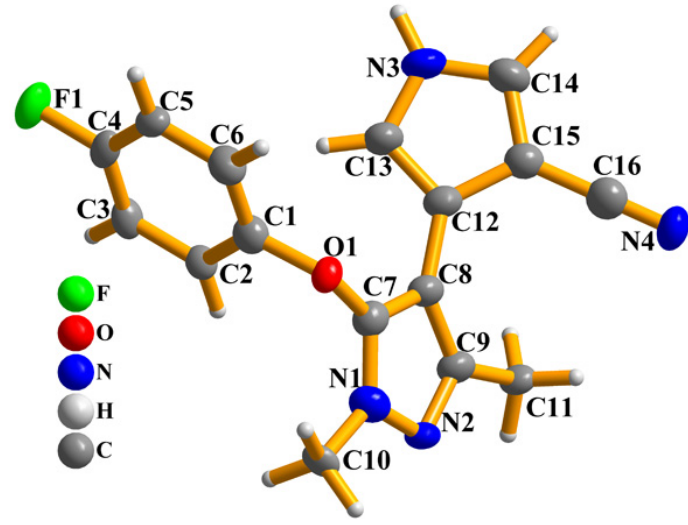

图 S27 11a 单分子结构

Figure S27 a single molecule structure of 11a

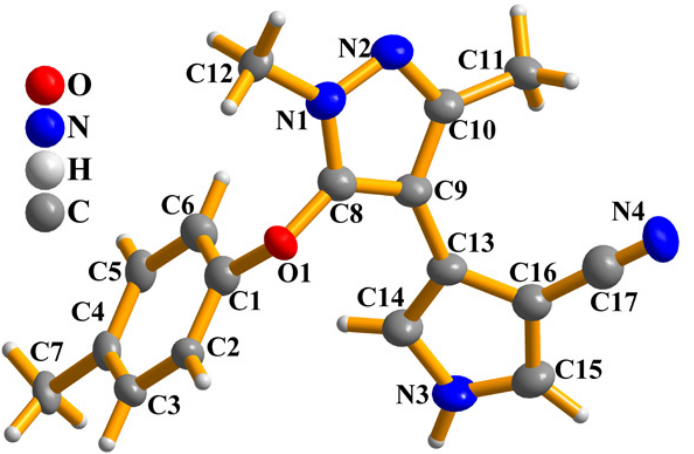

图 S28 11b 单分子结构

Figure S28 a single molecule structure of $\mathbf{1 1 b}$ 


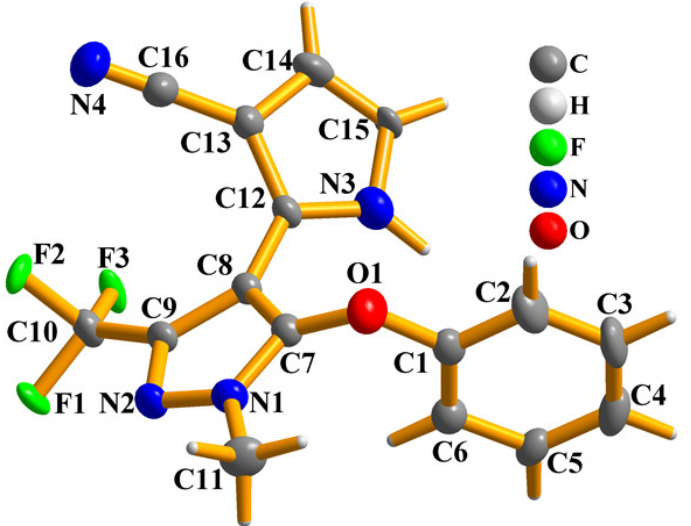

图 S29 11c 单分子结构

Figure S29 a single molecule structure of 11c

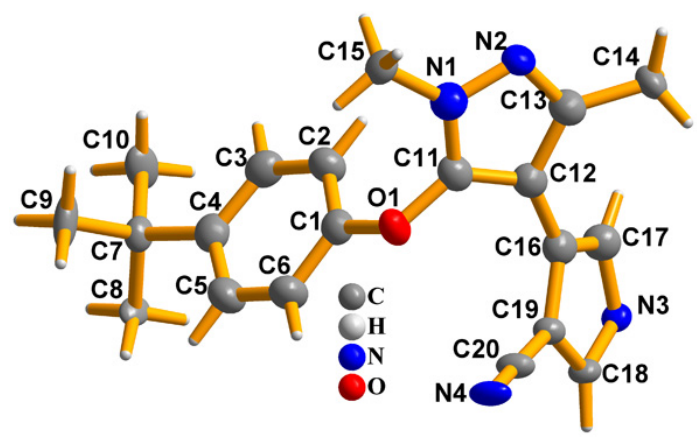

图 S30 11e 单分子结构

Figure S30 a single molecule structure of 11e

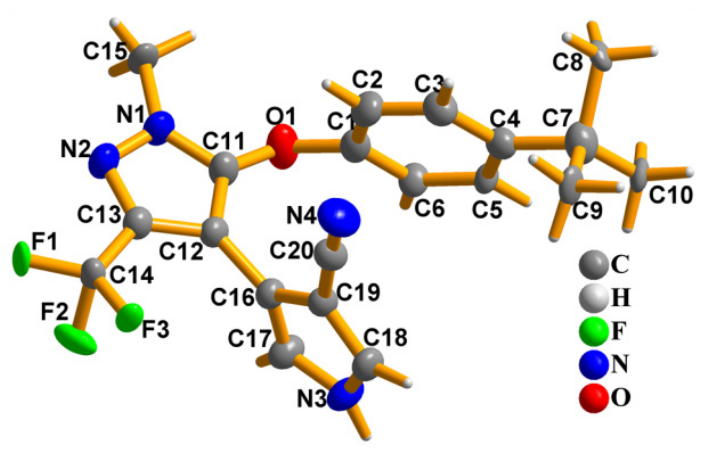

图 S31 11g 单分子结构

Figure S31 a single molecule structure of $\mathbf{1 1 g}$

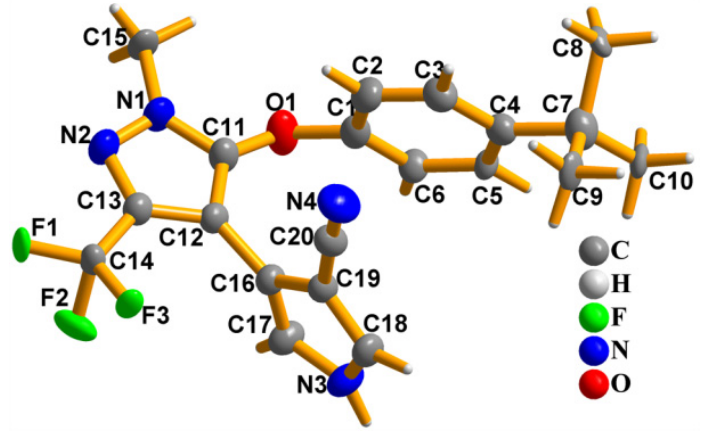

图 S32 11h 单分子结构

Figure S32 a single molecule structure of $\mathbf{1 1 h}$

\section{表 $\mathrm{S} 1$ 晶体 7a 7 $, 7 \mathrm{e}, 7 \mathrm{~g}, 7 \mathrm{~h}$ 的结构数据和精修参数}

Table S1 Crystal data and refinement parameter for 7a $\sim \mathbf{7 c}, 7 \mathrm{e}, \mathbf{7 g}, \mathbf{7 h}$

\begin{tabular}{lccc}
\hline Crystal parameters & $\mathbf{7 a}$ & $\mathbf{7 b}$ & $\mathbf{7 c}$ \\
\hline Empirical formula & $\mathrm{C}_{16} \mathrm{H}_{13} \mathrm{FN}{ }_{4} \mathrm{O}$ & $\mathrm{C}_{17} \mathrm{H}_{16} \mathrm{~N}_{4} \mathrm{O}$ & $\mathrm{C}_{20} \mathrm{H}_{24} \mathrm{~N}_{4} \mathrm{O}_{2}$ \\
Formula weight & 296.30 & 292.34 & 352.43 \\
Crystal system & Monoclinic & Monoclinic & Orthorhombic \\
Space group & $P 2{ }_{1} / \mathrm{c}$ & $P 2{ }_{1} / \mathrm{c}$ & $P 2{ }_{1} / \mathrm{c}$ \\
$a(\AA)$ & $11.8575(4)$ & $12.0871(4)$ & $12.1578(4)$ \\
$b(\AA)$ & $8.6032(3)$ & $8.5585(3)$ & $9.8584(4)$ \\
$c(\AA)$ & $17.6234(9)$ & $17.7768(9)$ & $17.0826(6)$ \\
$\alpha\left(^{\circ}\right)$ & 90 & 90 & 90 \\
$\beta\left(\left(^{\circ}\right)\right.$ & $125.441(3)$ & $121.968(3)$ & $100.343(3)$ \\
$\gamma\left({ }^{\circ}\right)$ & 90 & 90 & 90
\end{tabular}


$V\left(\AA^{3}\right)$

Z

$D_{\text {caled }}\left(\mathrm{Mg} \mathrm{cm}^{-3}\right)$

$\mu\left(\mathrm{mm}^{-1}\right)$

collected/ unique

$R_{\text {int }}$

GOF on $F^{2}$

$R(\mathrm{I}>2 \sigma(\mathrm{I}))$

$R$ (all data)
1464.70(10)

4

1.344

0.097

$5077 / 2565$

0.0202

1.039

${ }^{\mathrm{a}} \mathrm{R} 1=0.0402$

${ }^{\mathrm{b}}{ }_{\mathrm{wR}} 2=0.1037$

${ }^{\mathrm{a}} \mathrm{R} 1=0.0511$

${ }^{\mathrm{b}} \mathrm{wR} 2=0.1114$
$1560.7(11)$

4

1.245

0.081

$5832 / 2744$

0.0161

1.027

${ }^{\mathrm{a}} \mathrm{R}_{1}=0.0400$

${ }^{\mathrm{b}} \mathrm{wR}_{2}=0.1070$

${ }^{\mathrm{a}} \mathrm{R}_{1}=0.0503$

${ }^{\mathrm{b}} \mathrm{wR}_{2}=0.1158$
2014.19(13)

4

1.162

0.077

$7252 / 3546$

0.0159

1.034

${ }^{\mathrm{a}} \mathrm{R}_{1}=0.0511$

${ }^{\mathrm{b}} \mathrm{wR}_{2}=0.1387$

${ }^{\mathrm{a}} \mathrm{R}_{1}=0.0637$

${ }^{\mathrm{b}} \mathrm{wR}_{2}=0.1261$

${ }^{\mathrm{a}} \mathrm{R}_{1}=(\Sigma|| \mathrm{Fo}|-| \mathrm{Fc} \mid) / \Sigma|\mathrm{Fo}| \cdot{ }^{\mathrm{b}} \mathrm{wR}_{2}=\left[\Sigma \mathrm{w}\left(\mathrm{Fo}^{2}-\mathrm{Fc}^{2}\right)^{2} / \Sigma \mathrm{w}\left(\mathrm{Fo}^{2}\right)^{2}\right]^{1 / 2}$

\begin{tabular}{|c|c|c|c|}
\hline Crystal parameters & $7 e$ & $7 \mathrm{~g}$ & $7 \mathrm{~h}$ \\
\hline Empirical formula & $\mathrm{C}_{16} \mathrm{H}_{11} \mathrm{~F}_{3} \mathrm{~N}_{4} \mathrm{O}$ & $\mathrm{C}_{17} \mathrm{H}_{13} \mathrm{~N}_{4} \mathrm{O}$ & $\mathrm{C}_{20} \mathrm{H}_{24} \mathrm{~N}_{4} \mathrm{O}_{2}$ \\
\hline Formula weight & 332.29 & 345.31 & 388.39 \\
\hline Crystal system & Orthorhombic & Monoclinic & Orthorhombic \\
\hline Space group & $P b c a$ & $C 2 / \mathrm{c}$ & $P 2{ }_{1} / \mathrm{c}$ \\
\hline$a(\AA)$ & $8.7470(3)$ & $12.0871(4)$ & $8.7339(4)$ \\
\hline$b(\AA)$ & $18.4194(10)$ & $8.5585(3)$ & $13.4711(7)$ \\
\hline$c(\AA)$ & $19.4158(8)$ & $17.7768(9)$ & $17.5702(6)$ \\
\hline$\alpha\left(^{\circ}\right)$ & 90 & 90 & 90 \\
\hline$\beta\left(^{\circ}\right)$ & 90 & $121.968(3)$ & $95.716(4)$ \\
\hline$\gamma\left({ }^{\circ}\right)$ & 90 & 90 & 90 \\
\hline$V\left(\AA^{3}\right)$ & $3128.2(2)$ & $3333.5(3)$ & $2056.95(16)$ \\
\hline$Z$ & 8 & 8 & 4 \\
\hline$D_{\text {caled }}\left(\mathrm{Mg} \mathrm{cm}^{-3}\right)$ & 1.411 & 1.380 & 1.254 \\
\hline$\mu\left(\mathrm{mm}^{-1}\right)$ & 0.117 & 0.113 & 0.099 \\
\hline collected/ unique & $7211 / 2747$ & $5807 / 2940$ & $7340 / 3606$ \\
\hline$R_{\text {int }}$ & 0.0227 & 0.0205 & 0.0153 \\
\hline GOF on $F^{2}$ & 1.020 & 1.030 & 1.046 \\
\hline \multirow[t]{2}{*}{$R(\mathrm{I}>2 \sigma(\mathrm{I}))$} & ${ }^{\mathrm{a}} \mathrm{R} 1=0.0662$ & ${ }^{\mathrm{a}} \mathrm{R}_{1}=0.0438$ & ${ }^{\mathrm{a}} \mathrm{R}_{1}=0.0854$ \\
\hline & ${ }^{b}{ }_{w R} 2=0.1920$ & ${ }^{b} \mathrm{wR}_{2}=0.1071$ & ${ }^{b} \mathrm{wR}_{2}=0.1479$ \\
\hline \multirow[t]{2}{*}{$R$ (all data) } & ${ }^{\mathrm{a}} \mathrm{R} 1=0.0853$ & ${ }^{\mathrm{a}} \mathrm{R}_{1}=0.0615$ & ${ }^{\mathrm{a}} \mathrm{R}_{1}=0.0849$ \\
\hline & ${ }^{b}{ }_{w R 2}=0.2112$ & ${ }^{b}{ }_{w R} R_{2}=0.1204$ & ${ }^{b}{ }_{w R}=0.1686$ \\
\hline
\end{tabular}

